\title{
ONE ZONE MODELING OF IRREGULAR VARIABILITY OF STELLAR CONVECTIVE ENVELOPE
}

\author{
W. UNNO \\ Research Institute for Science and Technology, Kinki University, Kowakae, \\ Higasi-Osaka-shi, Osaka, 577 Japan \\ and \\ D.-R. XIONG \\ Purple Mountain Observatory, Academia Sinica, Nanjing, 6001, \\ Peoples' Republic of China
}

\begin{abstract}
One zone modeling of the irregular variability of red super-giants is intended with regard to the nonlinear coupling of finite amplitude pulsation with convection. The nonlocal mixing length is employed for the evaluation of the convective flux, the turbulent pressure and the turbulent power of temperature fluctuations. The radial pulsation and the Boussinesq convection are assumed for simplicity. The one zone is defined as the layer having the entropy maximum and the minimum at the bottom and at the top, respectively. The quasi-adiabatic approximation is consistent with this definition in fixing the zone to the same mass range. The spatial derivatives are evaluated under the assumption of homologous changes with the equilibrium homologous parameters. Then, a set of 6 simultaneous first order nonlinear ordinary differential equations are obtained as the one zone representation of the irregular variability of the convective envelope.
\end{abstract}

Key words: Irregular variability - Convection pulsation coupling - Nonlinear dynamical system - One zone modeling

\section{Introduction}

Stars are dynamically stable, and so are red giants. Because of deep convective envelopes, however, the stability of red giants is relatively weak, and the oscillation easily grows to a large amplitude with the significant driving kappa-mechanism. Saitou, Takeuti and Tanaka (1989) has shown in the one-zone treatment that the saturation of the diving power due to the largeamplitude dissipation leads to the semi-regular variability of yellow giants. An improved one-zone modeling for the irregular variability is intended in the present paper with full regards to the fundamental role of the convective envelope.

The radiation hydrodynamics has been formulated conveniently for the study of the nonlinear coupling between the convection and the pulsation (Xiong, 1977; 1989). For the qualitative study of the origin of the irregular variability, however, simplifying approximations such as the Boussinesq convection and the isotropic turbulence may well be introduced (Unno and Xiong, 1990: hereafter referred to as Paper 1). Perhaps, the intrinsic difficulty of the standard one-zone modeling in which the zone is fixed with respect to the mass element may be the Lagrangian shift of the zone of par- 
tial ionization which is crucial for the physics of the variability. In the present paper, we will define the one-zone boundaries instantaneously as the layer of the maximum and the minimum entropy. This definition of the one zone has the advantage of ensuring negligible shifts of the zone with respect to the mass element by the introduction of the quasi-adiabatic approximation which is suitable for the qualitative study.

The mathematical technique of the one-zone modeling is either setting boundary conditions as many as the number of different hinds of spatial derivatives or assuming homologous changes of variables with the equilibrium homology invariants. In the original one-zone modeling, Baker (1966) neglected all the spatial derivatives of the relative variations except for the relative luminosity change. In that case, the only boundary condition was the zero luminosity change at the lower boundary. Proper boundary conditions for the thick one-zone are approximately given by the asymptotic solutions of the linear pulsation in the upper atmosphere and the radiative interior (Okamoto and Unno, 1967). In the nonlinear treatment, however, the assumption of the homologous changes of variables within the convection zone is simpler and consistent with the one-zone modeling. In the latter case, however, since the equilibrium relative luminosity change is zero, the homologous change method is untenable for the luminosity variation and Baker's boundary condition should be employed.

In the present paper, nonhomologous changes due to the shock wave formation, for example, are disregarded. But, since the time scales of the oscillation and the convection can be comparable (Buchler, 1990) and convection contains intrinsic instability, there can be ample chance for the deep convection zone to oscillate chaotically. Further numerical studies are desired.

\section{Equations of Pulsation-Convection Coupling}

Equations governing radial pulsations in the convective envelope are given in Paper 1 as follows,

$$
\begin{aligned}
& \bar{\rho} D_{t}(1 / \bar{\rho})=r^{-2} \partial_{r}\left(r^{2} \bar{u}\right), \\
& \bar{\rho} D_{t} \bar{u}=-\partial_{r}\left(\bar{p}+\bar{p}_{t}\right)+r^{-2} \partial_{r}\left(r^{2} \mu_{t} \partial_{r} \bar{u}\right),
\end{aligned}
$$

and

$$
\bar{\rho} \bar{T} D_{t} \bar{S}=-r^{-2} \partial_{r}\left[r^{2}\left(\bar{F}_{R}+\bar{F}_{C}\right)\right]-\bar{\rho} D_{t} \bar{E}_{t}-\bar{p}_{t}\left(r^{-2} \partial_{r}\right)\left(r^{2} \bar{u}\right)+\mu_{t}\left(\partial_{r} \bar{u}\right)^{2},(3)
$$

where overbars indicate the horizontal average (radial oscillation!),

$$
D_{t}=\partial_{t}+\bar{u} \partial_{r}
$$




$$
\begin{array}{ll}
F_{R}=-C_{p} \kappa_{R}\left(\partial_{r} T\right) \quad \text { with } & \kappa_{R}=4 a c T^{3} /\left(3 C_{p}^{\prime} \bar{\kappa} \rho\right), \\
F_{C}=\rho\left(h^{\prime}+u^{\prime 2} / 2\right) u_{r}{ }^{\prime} . & h_{t}=E_{t}+\left(p_{t} / \rho\right)=(5 / 2) u^{\prime 2},
\end{array}
$$

and $\mu_{t}$ denotes the turbulent viscosity given by

$$
\mu_{t}=R_{\text {eff }}^{-1} \bar{\rho}\left(\overline{u^{\prime 2}} / 3\right)^{1 / 2} H_{p},
$$

$R_{\text {eff }}$ being the effective Reynolds number $\left(\approx 10\left[1+\left(\tau_{t} / \tau_{0}\right)^{2}\right]\right)$, and $H_{p}$ the pressure scale height.

The convection quantities $\overline{F_{C}}$ and $\overline{p_{t}}$ representing the coupling of pulsation with convection in the above equations are governed by the following equations (see Paper 1 for the derivation),

$$
\begin{aligned}
& \left(D_{t}+2 \mu_{t} k^{2}\right)\left(\overline{p_{t}} / \bar{\rho}\right)=(4 / 9)\left\{-\bar{p}_{t}\left[r^{-2} \partial_{r}\left(r^{2} \bar{u}\right)\right]+\alpha\left(g+D_{t} \bar{u}\right)\left(\bar{F}_{C}-\bar{F}_{K^{*}}\right)\right\} \\
& {\left[\bar{\rho} D_{t}+\left(\mu_{t}+\kappa_{t}+\kappa_{R}\right) k^{2}\right](H / \bar{\rho})=-(2 / 3) H\left(\partial_{r} \bar{u}\right)} \\
& \quad+(2 / 3) \rho_{T} G\left(g+D_{t} \bar{u}\right)-\bar{p}_{t}\left[\partial_{r}\left(\bar{h}+\bar{h}_{t}\right)-\bar{\rho}^{-1} \partial_{r}\left(\bar{p}+\bar{p}_{t}\right)\right]
\end{aligned}
$$

and

$$
\begin{aligned}
{\left[\bar{\rho} D_{t}\right.} & \left.+2\left(\kappa_{t}+\kappa_{R}\right) k^{2}+\left(C_{p, T}+1\right) \bar{\rho}\left(D_{t} \bar{T} / \bar{T}\right)\right](G / \bar{\rho}) \\
& =-2\left(C_{p} \bar{T}\right)^{-1} H\left[\partial_{r}\left(\bar{h}+\overline{h_{t}}\right)-\bar{\rho}^{-1} \partial_{r}\left(\bar{p}+\bar{p}_{t}\right)\right],
\end{aligned}
$$

where

$$
\begin{aligned}
& G=C_{p} \bar{\rho} T^{\prime 2} / \bar{T}, \\
& \rho_{T}=-(\partial \log \rho / \partial \log T)_{p}, \quad C_{p, T}=\left(\partial \log C_{p} / \partial \log T\right)_{p}, \\
& \overline{F_{K}}=(1 / 2) \overline{\rho u^{\prime 2} u_{r}}=-(1 / 2) \kappa_{t}\left[\partial_{r}\left(\overline{p_{t}} / \bar{\rho}\right)\right] .
\end{aligned}
$$

The turbulent conductivity $\kappa_{t}$ is taken to be equal to the turbulent viscosity $\mu_{t}, \kappa_{t}=\mu_{t}$ (cf. Xiong and Chen, 1992).

\section{The One-Zone Modeling}

For irregular variability which depends critically on the parameters of the system, one-zone modeling is dispensable for overall qualitative studies. We define the one-zone convection model by the zone of decreasing entropy with height so that the entropy is maximum at the base and minimum at the top. The technique of the one-zone modeling lies in the replacement of the spatial derivatives with the corresponding ratios of the physical quantities. Thus, the only independent variable of the system will be the time $t$. To 
achieve this goal, we first transform the variables from the $(t . r)$ coordinate system to the $(t, \bar{S})$ coordinate system to write down the above system of equations. We write $s$ instead of $\bar{S}$ hereafter for brevity. We also employ the quasi-adiabatic approximation, $D_{t} \bar{S}=0$, in the transformation except in the energy equation. Then, the time-derivative of a quantity $Q, D_{t} Q$, in the $(t, r)$-representation is simply described by $d_{t} Q$ in the $(t, s)$-representation. Also, since the convection zone in the above definition is occupied by the same material, the assumption of the homologous variation within the zone reduces the spatial derivative, $\partial_{r} Q$, to $\left[Q_{r}\right](Q / r)$ in which $\left[Q_{r}\right]$ denotes the homology invariant,

$$
\left[Q_{r}\right]=\partial \log Q / \partial \log r
$$

The homology invariants are taken to be the same as the equilibrium value by assumption. For instance, we have

$$
\begin{aligned}
& r^{-2} \partial_{r}\left(r^{2} \bar{u}\right)=3(\bar{u} / r), \\
& \partial_{r} p=-V(p / r) \quad \text { with } \quad V=\left(G M_{r} \rho / r p\right)_{e q},
\end{aligned}
$$

and

$$
\partial_{r} \bar{p}_{t}=-(1 / 3)[4+(1-\nabla) V]\left(\bar{p}_{t} / r\right) \text { with } \nabla=(\partial \log T / \partial \log p) .
$$

An approximate relation, $r^{2} \overline{\rho u^{\prime 3}}=$ const., has been used to derive the above $\left[\left(\bar{p}_{t}\right)_{r}\right]$ value.

In the energy equation, however, neither the quasi-adiabatic approximation nor the homology approximation are obviously applicable. Baker's boundary condition that the luminosity $L$ is constant in time, $L_{\varepsilon q}$, at the base of the zone (Baker, 1966; Saitou et al., 1990) so that

$$
\left(\bar{\rho} r^{2}\right)^{-1} \partial_{\tau} L=2\left(L-L_{e q}\right) / m
$$

$m$ being the mass of the zone, should be used. The application of the above procedures of the one-zone modeling to the set of equations given in the last section yields the required one-zone equations given in the following section.

\section{Summary}

The results are summarized below. Omitting overbars from the symbols, we obtain the following set of one-zone equations,

$$
\begin{aligned}
& \rho d_{t}(1 / \rho)=3(u / r), \\
& \rho d_{t} u=V(p / r)-V_{t}\left(p_{t} / r\right)-\rho g-{k_{0}}^{2}\left(\mu_{t} u / r^{2}\right),
\end{aligned}
$$

where 


$$
V_{t}=(V / 3)(1-\nabla) \text { and } k_{0}^{2}=(V / 3)(2+\nabla)-4,
$$

for the equilibrium model by the mixing-length theory, and

$$
\begin{aligned}
C_{p}\left[d_{t} T\right. & \left.-\nabla_{a d}(T / p) d_{t} p\right] \\
= & -2\left(L-L_{\epsilon q}\right) / m-(3 / 2 \rho)\left[d_{t} p_{t}+5 p_{t} u / r\right]+\left(\mu_{t} / \rho\right)(u / r)^{2},
\end{aligned}
$$

where

$$
\begin{aligned}
& L=4 \pi r^{2}\left(F_{R}+F_{C}\right), \quad F_{R}=-(16 V \nabla / 3)\left(a c T^{4} / \bar{\kappa} \rho r\right), \\
& F_{C}=H+F_{K}, \quad \text { and } \quad F_{K}=-(V \nabla / 3)\left(\kappa_{t} p_{t} / r \rho\right) .
\end{aligned}
$$

The convection variables $p_{t}, H$, and $G$ are governed by the following set of one-zone convection equations,

$$
\begin{aligned}
& {\left[d_{t}+2 \mu_{t}\left(\pi / l_{e}\right)^{2}\right]\left(p_{t} / \rho\right)=(4 / 9)\left[-3 p_{t}(u / r)+\rho_{T}(\rho / T)\left(g+d_{t} u\right) H\right],} \\
& {\left[\rho d_{t}+\left(\mu_{t}+\kappa_{t}+\kappa_{R}\right)\left(\pi / l_{e}\right)^{2}\right](H / \rho)=-(2 / 3) H(u / r)} \\
& \quad+(2 / 3) \rho G\left(g+d_{t} u\right)+p_{t}\left[C_{p}(T / r) V\left(\nabla-\nabla_{a d}\right)-6 V_{t}\left(p_{t} / \rho r\right)\right],
\end{aligned}
$$

and

$$
\begin{aligned}
{\left[\rho d_{t}+\right.} & \left.2\left(\kappa_{t}+\kappa_{R}\right)\left(\pi / l_{e}\right)^{2}+\left(C_{p, T}+1\right) \rho\left(d_{t} T / T\right)\right](G / \rho) \\
& =-2\left(C_{p} T\right) H\left[C_{p}(T / r) V\left(\nabla-\nabla_{a d}\right)-6 V_{t}\left(p_{t} / \rho r\right)\right],
\end{aligned}
$$

where $l_{e}$ denotes the mixing length $(\simeq$ scale height $)$.

\section{References}

Baker, N.H.: 1966, in Stellar Evolution, eds. R.F.Stein and A.G.F. Cameron (Prenum Press, New York), p. 333.

Buchler, J.R.: 1990, in Nonlinear Astrophysical Fluid Dynamics, Annals New York Acad. Sci., 617, 17.

Saitou, M., Takeuti, M., and Tanaka, Y.: 1989, Publications of the ASJ 41, 297.

Unno, W. and Xiong, D.-R.: 1990, in Progress of Seismology of the Sun and Stars, eds. Y. Osaki and H. Shibahashi (Springer-Verlag), p. 103.

Xiong, D.-R.: 1977, Acta Astronomica Sinica 18, No.1, 26.

Xiong, D.-R.: 1989, Astronomy and Astrophysics 209, 126.

Xiong, D.-R. and Chen, J.-J.: 1992, Astronomy and Astrophysics, in press. 